\title{
Doppler Effect-Based Automatic Landing Procedure for UAV in Difficult Access Environments
}

\author{
Jan M. Kelner and Cezary Ziółkowski \\ Institute of Telecommunications, Faculty of Electronics, Military University of Technology, Gen. Sylwester Kaliski Str. No. 2, \\ 00-908 Warsaw, Poland \\ Correspondence should be addressed to Jan M. Kelner; jan.kelner@wat.edu.pl
}

Received 23 June 2017; Accepted 14 August 2017; Published 4 October 2017

Academic Editor: Seungjae Lee

Copyright (c) 2017 Jan M. Kelner and Cezary Ziółkowski. This is an open access article distributed under the Creative Commons Attribution License, which permits unrestricted use, distribution, and reproduction in any medium, provided the original work is properly cited.

Currently, almost unrestricted access to low-lying areas of airspace creates an opportunity to use unmanned aerial vehicles (UAVs), especially those capable of vertical take-off and landing (VTOL), in transport services. UAVs become increasingly popular for transporting postal items over small, medium, and large distances. It is forecasted that, in the near future, VTOL UAVs with a high take-off weight will also deliver goods to very distant and hard-to-reach locations. Therefore, UAV navigation plays a very important role in the process of carrying out transport services. At present, during the flight phase, drones make use of the integrated global navigation satellite system (GNSS) and the inertial navigation system (INS). However, the inaccuracy of GNSS + INS makes it unsuitable for landing and take-off, necessitating the guidance of a human UAV operator during those phases. Available navigation systems do not provide sufficiently high positioning accuracy for an UAV. For this reason, full automation of the landing approach is not possible. This paper puts forward a proposal to solve this problem. The authors show the structure of an autonomous system and a Doppler-based navigation procedure that allows for automatic landing approaches. An accuracy evaluation of the developed solution for VTOL is made on the basis of simulation studies.

\section{Introduction}

Military applications [1-3] were the beginning of the development of unmanned aircraft systems. The increase in their availability contributes to the widespread use of unmanned aerial vehicles (UAVs) in civilian applications. In this case, monitoring large areas of land or sea is the main purpose. It concerns such areas of human activity as an agriculture $[4,5]$, energetics (i.e., photovoltaic plants [6-8] and high voltage lines [9]), environment protection [10], search and rescue [11], forestry and fire detection $[12,13]$, water area management $[14,15]$, and so on. An automation of monitoring procedures, low costs, and minimization of human resources in the UAV exploitation are conducive to the dynamic growth of their use in the civilian applications. The UAV monitoring is mainly based on optical sensors, whereas take-off and landing are usually done in the same place. Currently, almost unrestricted access to low-lying areas of airspace creates an opportunity to use UAVs in transport services. In this case, the place of the take-off and landing is far removed. This significantly hinders the implementation of navigation procedures, especially at the landing stage. In this article, we present a method of automatic landing approach that can be used especially in UAV transport services over long distances.

The advantage of this mode of transport is its independence of road infrastructure, traffic volume, and difficult terrain conditions. Hence, UAVs are increasingly used to transport long distances to small postal items and medicines in hard-to-reach areas. A practical example of such a solution, developed at the initiative of the United Nations Children's Fund (UNICEF), is the use of UAVs to transport blood samples in Malawi (Africa) [16]. Nowadays, the transportation of blood at close distance between hospitals [17] or other packages [18] is already achieved. In the future, increasing the load capacity of UAVs will enable fast transport services in hard-to-reach environments such as islands, mountainous, desert, and polar areas (i.e., the Arctic and the Antarctic). In this case, vertical take-off and landing (VTOL) UAVs will play a special role, as they do not require landing strips, but only small landing pads. Hence, VTOLs may be used to land at 
such locations as islands, oil platforms, vessels, or skyscraper roofs.

The basic method for navigating UAVs over long distances is based on the use of a global navigation satellite system (GNSS) supported by the UAV's own inertial navigation system (INS) [19-22]. However, these systems cannot be directly used in the final stage of the flight, that is, during landing, due to the low positioning accuracy of moving objects inherent in such systems. A practical solution to this problem is to use optical cameras, which allow the operator to remotely control the landing process. However, this method requires the use of a broadband control channel and may only be used during daylight and in good visibility conditions. Under night conditions and in poor visibility, a thermal imaging camera $[6,23-25]$ or synthetic aperture radar (SAR) [26-28] may be used. Furthermore, long distance wireless communications are characterized by significant delays and, in the case of the hard-to-reach areas, only satellite communications [29] can be used. In these cases, the navigation system requires a large and expensive extension, which prevents its commercial use. High costs and, above all, the necessity to change the destination location (landing area) for the transport also prevent the use of conventional landing navigation systems $[20,21,30,31]$ such as the instrument landing system (ILS), microwave landing system (MLS), or local area augmentation system (LAAS). In this case, the operational range for these systems is limited to the space around large airports.

The high precision required for determining the current position of the object and the required flexibility in terms of landing spots limit the ability to use UAVs for air transport. Therefore, the study of a precise and simple positioning method, which would give the user flexibility in terms of landing locations and conditions, is essential for development of this transport sector. This paper presents a proposal for a solution that uses spatially distinctive features of the Doppler effect. The presented navigation procedure is based on an analytical relationship that describes the Doppler frequency shift (DFS), as a function of the receiver position [32]. This formula is the basis of the signal processing method called the signal Doppler frequency (SDF) [33-35], which is used in the location systems of emission sources [36] and navigation of objects $[37,38]$. Using this method enables complete automation of the UAV landing procedure and eliminates the need for a broadband remote control channel. Contemporary navigational systems often make use of pulse signals. Consequently, these systems require large spectrum resources. In contrast to these solutions, the developed system is based on narrowband signals (harmonics), which minimizes the spectrum cost. Simulation studies for VTOL are performed in order to determine the effectiveness of the developed procedure, that is, the accuracy with which the flight trajectory is determined. The obtained results show the position errors that may occur during the VTOL landing process and thus provide the opportunity to evaluate the practical implementation of the developed procedure.

The remainder of the paper is organized as follows. Section 2 presents operation principles of the Doppler-based landing approach system for UAVs. The authors show a structure of this system and a navigation procedure for the landing phase. Simulation scenarios are described in Section 3. In simulation studies, the authors assume that the vehicle is VTOL-capable. In Section 4, obtained results are presented. Section 5 contains the summary of the paper.

\section{Operation Principle of the Proposed Landing System}

Assuming that the receiver (i.e., the UAV) moves at constant velocity, $v$, the relationship between DFS, $f_{D}$, and the signal source coordinates, $(x, y, z)$, is described by [32]

$$
f_{D}(x, y, z, t) \cong f_{D \max } \frac{x-v t}{\sqrt{(x-v t)^{2}+y^{2}+z^{2}}},
$$

where $f_{D \max }=f_{0} v / c$ is the maximum DFS, $f_{0}$ is the carrier frequency of the emitted signal, and $c$ is the speed of light.

Based on (1), it follows that, for known $(x, y, z)$, direction, and values of $v$, DFS measurement gives the possibility of determining the coordinates of two possible positions of the receiver. By using a system of two reference sources that are located at the distance $r$, we eliminate the ambiguity of the result. Averaging the results obtained from several reference sources reduces the error of estimation for the position coordinates of the object. Thus, increasing the number of reference sources increases the accuracy of the positioning of the receiver. The structure of the narrowband automatic landing system based on the SDF method is shown in Figure 1.

The basic elements of the system are the narrowband navigation receiver (NR) and GNSS receiver integrated with INS, both of which are installed in the UAV, and four radio beacons (RBs) that serve as reference signal sources. Three of RBs emit harmonic signals at frequencies $f_{1}, f_{2}$, and $f_{3}$, respectively. The fourth $\mathrm{RB}$ emits a modulated signal that contains information about the location of each RB relative to the destination UAV landing site. In addition, the narrowband measurement receiver (MR) of this $R B$ measures the current frequency of each RB. This information is also included with the modulating signal. This minimizes the impact of RB instability on the DFS determination accuracy [39]. The frame structure of the modulating signal is presented in Figure 2. Transmission of information contained in the modulation signal frame is based on differential binary phase shift keying (DPSK). Selecting this modulation type makes it easy to eliminate signal modulation. The operation of raising the DPSK signal to the second power provides for the reconstruction of the carrier wave whose frequency includes DFS [40].

As shown in Figures 1 and 4, the UAV navigation process consists of two essential stages: long-range navigation and landing. Long-range navigation uses integrated GNSS + INS, because at this flight stage, accurate positioning is not a critical issue. However, the landing stage requires high positioning accuracy. The standard GNSS receiver cannot achieve such accuracy, and thus navigation stage requires the use of dedicated solutions. A generalized algorithm of the precise UAV positioning at the landing stage is shown in Figure 3. 


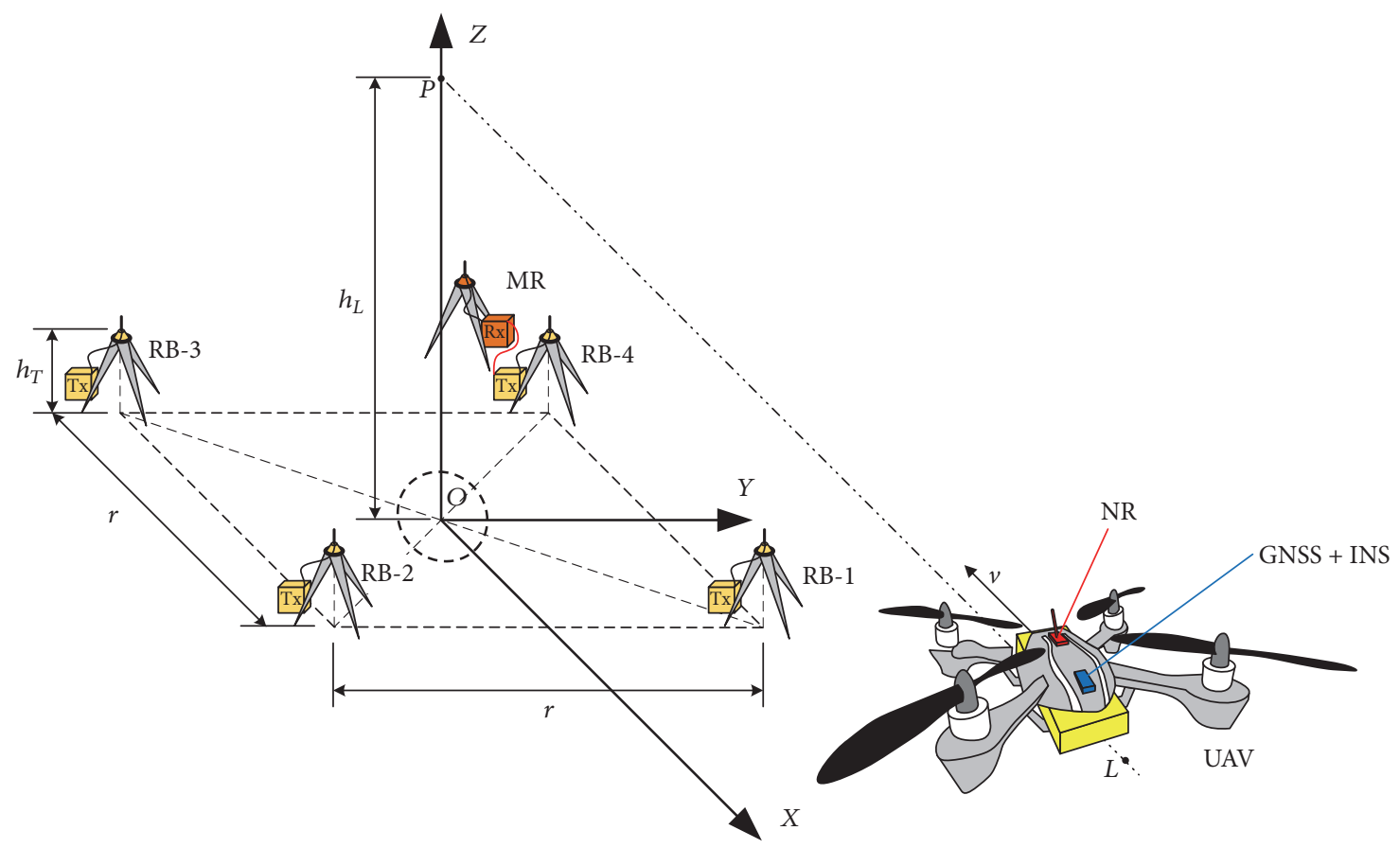

FIGURE 1: Structure of the automatic landing system.

Current frequencies of each RB from MR

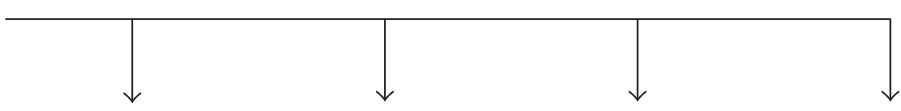

\begin{tabular}{|c|c|c|c|c|c|c|c|c|c|}
\hline Frame Index & {$\left[\begin{array}{lllll}X & Y & Y & Z & Z\end{array}\right]$} & $F 1$ & {$\left[\begin{array}{llll}X & Y & 2 & Z 2\end{array}\right]$} & $F 2$ & {$\left[\begin{array}{lllll}X & X & Y & Z & Z\end{array}\right]$} & $F 3$ & {$\left[\begin{array}{lllll}X & Y & 4 & Z\end{array}\right]$} & $F 4$ & $\begin{array}{c}\text { Cyclic Redundancy Check } \\
\text { (CRC) }\end{array}$ \\
\hline & RB-1 & & RB-2 & & RB-3 & & RB-4 & & \\
\hline
\end{tabular}

FIGURE 2: Frame structure of the modulating signal.

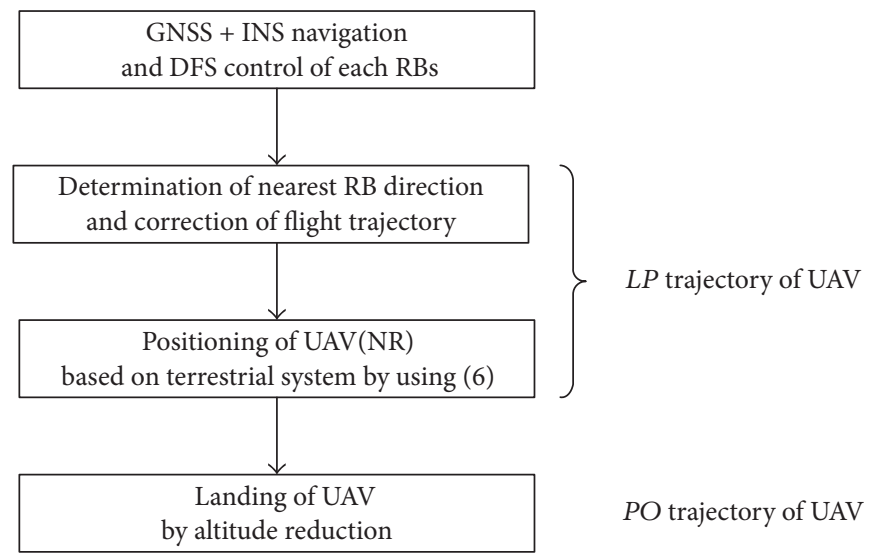

FIGURE 3: Generalized algorithm of the precise UAV positioning at the landing stage. 


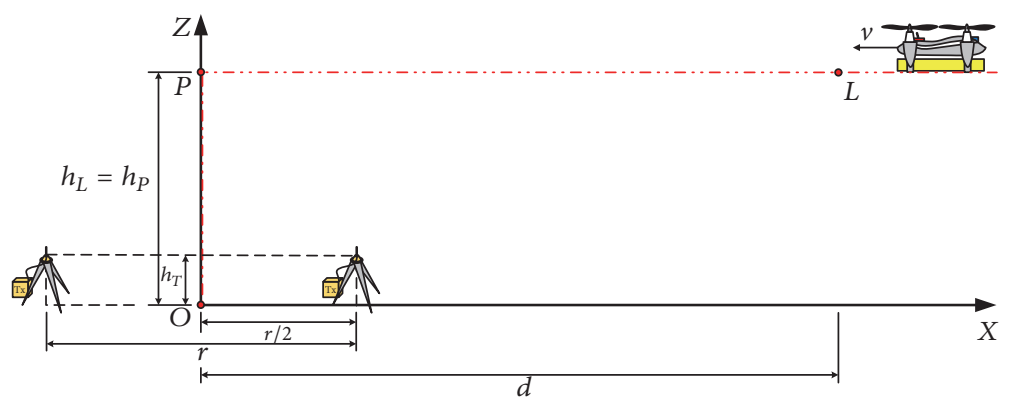

(a)

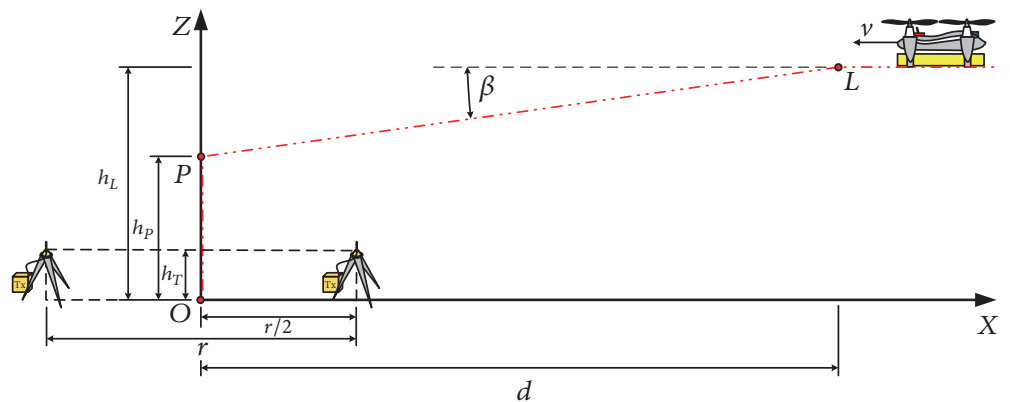

(b)

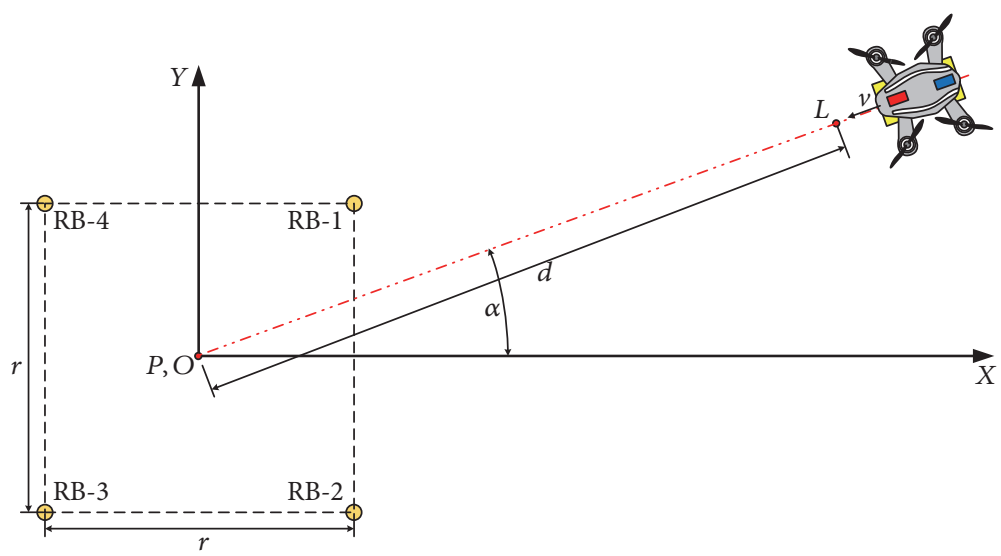

(c)

FIGURE 4: Direction of VTOL arrival to landing site with respect to the location of RBs.

Strictly determined DFSs, which occur for a close proximity to particular RBs, are the criterion for the transition of the UAV navigation system to the landing stage (point $L$, see Figures 1 or 4). At a relatively large distance from the landing site, the azimuth angle, $\varphi$, has a dominant impact on $f_{D}$. In this case, it is

$$
\theta \cong \varphi, \text { so } f_{D} \cong f_{D \max } \cos \varphi,
$$

where $\theta$ is the angle between the velocity vector of the receiver (i.e., UAV) and the direction to the signal source (i.e., RB).

Hence, changes of DFSs on the LP (see Figures 1 or 4) line segment are the basis for correcting the UAV flight direction at a fixed altitude. Length of this segment is $d$. Because UAV is moving in the direction of signal sources, the criterion of this correction is a simultaneous maximization of DFSs for the all RBs' signals. In close proximity, the elevation angle begins to play a significant impact. If the smallest DFSs decrease to a certain criteria value, for example, $0.8 f_{D \max }$, then UAV changes the flight direction by $\theta$ and begins to approach toward the nearest RB. During movement, the RBs' coordinates are determined in NR with respect to the system whose $O$ (see Figures 1 or 4 ) is the origin and the $x$ axis coincides with the direction of the new trajectory. The formulas that describe the RBs' coordinates are defined using the SDF method [33]

$$
\begin{gathered}
\tilde{x}_{k}=v \frac{t_{2} A_{k}\left(t_{2}\right)-t_{1} A_{k}\left(t_{1}\right)}{t_{2} A_{k}\left(t_{2}\right)-t_{1} A_{k}\left(t_{1}\right)}, \quad \forall_{k=1, \ldots, K} \\
\tilde{y}_{k}^{2}+\widetilde{z}_{k}^{2}=\left[v \frac{\left(t_{2}-t_{1}\right) A_{k}\left(t_{2}\right) A_{k}\left(t_{1}\right)}{A_{k}\left(t_{2}\right)-A_{k}\left(t_{1}\right)}\right]^{2}, \quad \forall_{k=1, \ldots, K},
\end{gathered}
$$


where $\left(\widetilde{x}_{k}, \tilde{y}_{k}, \widetilde{z}_{k}\right)$ is the estimated coordinates of the $k$ th $\mathrm{RB}$, $A_{k}(t)=\sqrt{1-F_{k}^{2}(t)} /\left|F_{k}(t)\right|, F_{k}(t)=\left[c \tilde{f}_{D k}(t)\right] /\left[v f_{k}(t)\right]$, $\tilde{f}_{D k}(t)$ is DFS for the $k$ th RB estimated by NR at $t$ time moment, $f_{k}(t)$ is the carrier frequency of the $k$ th RB signal measured by MR and obtained from the last received data frame, and $t_{1}$ and $t_{2}$ are two different time moments of the DFS measurement.

The navigational coordinates of UAV are determined by transforming the RBs' coordinates and the coordinates obtained by the system based on the UAV's flight trajectory

$$
\begin{aligned}
& \tilde{x}_{\mathrm{UAV} k}=x_{k}+\left(\tilde{x}_{k} \cos \widetilde{\alpha}+\tilde{y}_{k} \sin \widetilde{\alpha}\right) \cos \tilde{\beta}+\widetilde{z}_{k} \sin \tilde{\beta}, \\
& \forall_{k=1, \ldots, K} \\
& \tilde{y}_{\mathrm{UAV} k}=y_{k}-\tilde{x}_{k} \sin \widetilde{\alpha}+\tilde{y}_{k} \cos \widetilde{\alpha}, \quad \forall_{k=1, \ldots, K} \\
& \tilde{z}_{\mathrm{UAV} k}=z_{k}-\left(\widetilde{x}_{k} \cos \widetilde{\alpha}+\tilde{y}_{k} \sin \widetilde{\alpha}\right) \sin \widetilde{\beta}+\widetilde{z}_{k} \cos \widetilde{\beta} \text {, } \\
& \forall_{k=1, \ldots, K},
\end{aligned}
$$

where $\left(\widetilde{x}_{k}, \tilde{y}_{k}, \widetilde{z}_{k}\right)$ are the real coordinates of the $k$ th $\mathrm{RB}$ included in each data frame and $\widetilde{\alpha}$ and $\widetilde{\beta}$ are the estimated directions of the UAV flight in the azimuth $(O X Y)$ and elevation $(O X Z)$ planes determined relative to the destination landing site (see Figure 4 ) by using (3); that is,

$$
\begin{aligned}
& \tilde{\alpha}=\operatorname{atan}\left(\frac{1}{K} \sum_{k=1}^{K} \frac{\tilde{y}_{k}+y_{k}}{\tilde{x}_{k}+x_{k}}\right) \\
& \widetilde{\beta}=\operatorname{atan}\left(\frac{1}{K} \sum_{k=1}^{K} \frac{\widetilde{z}_{k}+z_{k}}{\tilde{x}_{k}+x_{k}}\right) .
\end{aligned}
$$

In $[33,35,37]$, analysis of the SDF method shows that the trajectory location relative to the signal source has a significant influence on the accuracy of the positioning the object. The smallest positioning error occurs when $\alpha$ tends to $90^{\circ}$, that is, when DFS converges to zero. Therefore, to minimize the navigation error, the weighted average coordinates relative to the individual RBs are used to estimate the UAV coordinates [37]

$$
\begin{aligned}
& \left(\tilde{x}_{\mathrm{UAV}}, \tilde{y}_{\mathrm{UAV}}, \widetilde{z}_{\mathrm{UAV}}\right) \\
& =\left(\frac{1}{W} \sum_{k=1}^{K} w_{k} \widetilde{x}_{\mathrm{UAV} k}, \frac{1}{W} \sum_{k=1}^{K} w_{k} \widetilde{y}_{\mathrm{UAV} k}, \frac{1}{W} \sum_{k=1}^{K} w_{k} \widetilde{z}_{\mathrm{UAV} k}\right),
\end{aligned}
$$

where $w_{k}=1-\left|F_{k}(t)\right|$ and $W=\sum_{k=1}^{K} w_{k}$.

RB system simplicity gives us the ability to position the navigation system in any field conditions. Additionally, the minimization of spectral resources is a significant advantage of the presented method compared to the existing solutions.

\section{Scenarios of Simulation Studies}

The effectiveness of the developed navigation procedure determines the accuracy of determining the current UAV coordinates. In this paper, an assessment of the procedure accuracy is made on the basis of simulation tests, of which scenarios concern the VTOL navigation. In our studies, the following assumptions and input data are accepted:

(i) landing point is the origin of the coordinate system;

(ii) base of the navigation system is four RBs whose positions describe the coordinates, $\left(x_{k}, y_{k}, z_{k}\right)$, where $k=1,2, \ldots, K,(K=4)$; the RBs' coordinates and frequencies, $f_{k}$, of the emitted signals are presented in Table 1;

(iii) the Kth RBs emit the modulated DPSK signal, which contains information about the position coordinates and the current frequencies of the individual RBs;

(iv) bandwidth of the DPSK signal is $B_{T}=80 \mathrm{kHz}$;

(v) operating frequency of NR is $f_{R}=2.4 \mathrm{GHz}$ and the reception bandwidth is about $B_{R}=500 \mathrm{kHz}$;

(vi) instantaneous DFS is determined every $0.5 \mathrm{~s}$ on the basis of the spectral analysis duration of $1.0 \mathrm{~s}$; basic frequency of the spectral analysis is $0.1 \mathrm{~Hz}$;

(vii) to analyze the Doppler curves, the time windows $T_{1}=$ $5 \mathrm{~s}$ and $T_{2}=10 \mathrm{~s}$ are used;

(viii) in electromagnetic environment, additive noise is occurred, and the level of the emitted signals at the most distant point of the trajectory provides SNR = $8 \mathrm{~dB}$;

(ix) VTOL speed is $v=72 \mathrm{~km} / \mathrm{h}=20 \mathrm{~m} / \mathrm{s}$ and the flight altitude is $h_{L}=50 \mathrm{~m}$.

The research focuses on the impact assessment of various factors, such as the VTOL trajectory position and the flight direction relative to the RBs. In the first stage of simulation studies, two scenarios, Sc. 1 and Sc. 2 , are examined $(\alpha=0)$. Figure 4 shows their geometry in the elevation ( $\mathrm{a}$ and $\mathrm{b}$, resp., for Sc. 1 and Sc. 2) and azimuth plane (c). The second stage of the research is also based on Sc. 1 and Sc. 2. In this case, the study focuses on the impact assessment of the arrival direction in the azimuth plane, $\alpha$.

We assumed that the UAV is flying using the long-range navigation (GNSS + INS) method at an average altitude of $h_{L}$. At the point $L$, the aircraft navigation system switches to the landing phase, that is, it begins to use the navigation procedure descripted in Section 2. In Sc. 1, VTOL flies at a constant altitude to the point $P$ located above the landing site. The destination landing point $O$ is reached by reducing the altitude in the vertical direction. In the case of Sc. 2 , the flight on the line segment $L P$ is performed at the angle $\beta$, assuming that, at the point $P, \mathrm{UAV}$ is at the altitude $h_{P}$. This angle is determined by

$$
\beta=\operatorname{atan}\left(\frac{h_{L}-h_{P}}{d}\right),
$$

where $d$ is the length of $L P$ graphical projected on the azimuth plane $(O X Y)$. In simulation studies, we assume that $d=$ $400 \mathrm{~m}$ and $h_{P}=30 \mathrm{~m}$.

The VTOL approach direction with respect to the position of RBs has also a significant influence on the navigation 
TABLE 1: Location coordinates of RBs and frequencies of signals emitted by them.

\begin{tabular}{lccccc}
\hline$k$ th RB & $x_{k}(\mathrm{~m})$ & $y_{k}(\mathrm{~m})$ & $z_{k}=h_{T}(\mathrm{~m})$ & $f_{k}(\mathrm{kHz})$ & Notes \\
\hline 1 & 20 & 20 & 2 & $2,399,800$ & Harmonic signal \\
2 & 20 & -20 & 2 & $2,399,850$ & Harmonic signal \\
3 & -20 & -20 & 2 & $2,399,900$ & Harmonic signal \\
4 & -20 & 20 & 2 & $2,400,100$ & DPSK; RB + MR \\
\hline
\end{tabular}

accuracy. The results of these studies show the required location of RBs relative to the expected direction of VTOL approach. In simulation studies, Sc. 1 and Sc. 2 are also used to evaluate the impact of the VTOL approach direction on the navigation error.

\section{Results}

The simulation studies involve the implementation of procedures such as the generation of RBs' harmonic signals, generation of environmental noise, and determination of the VTOL coordinates based on the estimated DFSs. Each generated harmonic signal contains DFS that results from the VTOL position relative to corresponding RB. This DFS is determined on the basis of (1). The generated environmental noise is a normal band signal, whose dynamics relative to the harmonic signal dynamics provides $\mathrm{SNR}=8 \mathrm{~dB}$ at the range of the navigation system. The VTOL positioning procedure is performed as described in Section 2.

To evaluate the positioning error, $\Delta R$, also called the navigation error, the following metric is used:

$$
\begin{aligned}
& \Delta R \\
& =\sqrt{\left(\widetilde{x}_{\mathrm{UAV}}-x_{\mathrm{UAV}}\right)^{2}+\left(\tilde{y}_{\mathrm{UAV}}-y_{\mathrm{UAV}}\right)^{2}+\left(\widetilde{z}_{\mathrm{UAV}}-z_{\mathrm{UAV}}\right)^{2}},
\end{aligned}
$$

where $\left(\tilde{x}_{\mathrm{UAV}}, \tilde{y}_{\mathrm{UAV}}, \tilde{z}_{\mathrm{UAV}}\right)$ and $\left(x_{\mathrm{UAV}}, y_{\mathrm{UAV}}, z_{\mathrm{UAV}}\right)$ are the estimated and real coordinates of UAV, respectively.

The assessment of the impact of the landing trajectory position with respect to $\mathrm{RBs}$ is performed for $\alpha=0^{\circ}$ and two analyzed temporal windows. Figures 5 and 6 present the navigation error versus the distance to the target landing site for $T_{1}$ and $T_{2}$, respectively.

As you can see, the average navigation errors, $\overline{\Delta R}$ on line section $d=400 \mathrm{~m}$ for Sc. 1, are more than 1.3 and 1.6 times smaller compared to Sc. 2 for $T_{1}$ and $T_{2}$, respectively. At the target landing point, these errors reach $T_{1}$ Sc. $1: \overline{\Delta R}=0.08 \mathrm{~m}$ and Sc. $2: \overline{\Delta R}=0.33 \mathrm{~m}$; on the other hand $T_{2}$ Sc. $1: \overline{\Delta R}=$ $0.18 \mathrm{~m}$ and Sc. $2: \overline{\Delta R}=0.46 \mathrm{~m}$. For Sc. 2 , the navigation error has a large deviation (spread), $\sigma_{\Delta}$, which is $12 \mathrm{~m}$, and for Sc. 1 , this error deviation does not exceed $5 \mathrm{~m}$.

The direction of the VTOL landing approach relative to the RBs' locations has also a significant impact on $\Delta R$. Based on Sc. 1 for $T_{1}$, simulation studies that show a variability in navigation accuracy as a function of distance from the landing site are performed. The results of these tests, that is, $\Delta R$ versus the distance from the landing point, are shown in Figure 7 for selected values $\alpha=0^{\circ}, \alpha=20^{\circ}$, and $\alpha=40^{\circ}$.

It can be seen that the proper VTOL approach direction with respect to the landing site minimizes navigation

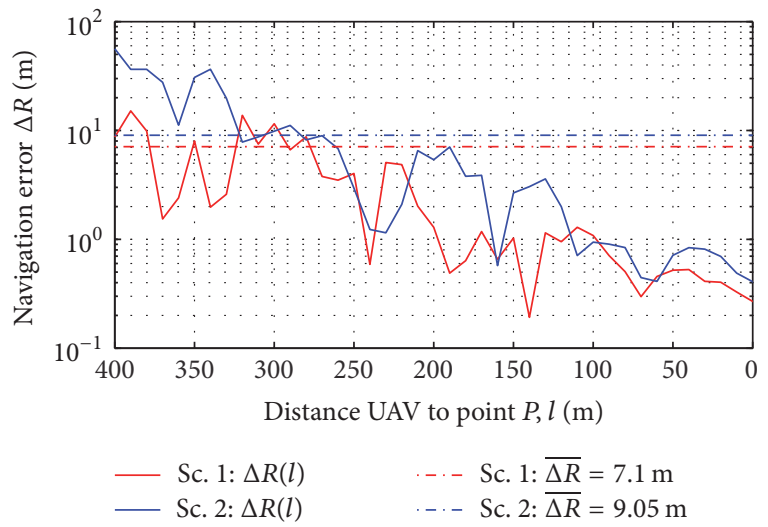

FIgURE 5: Navigation error for Sc. 1 and Sc. 2 and $T_{1}$.

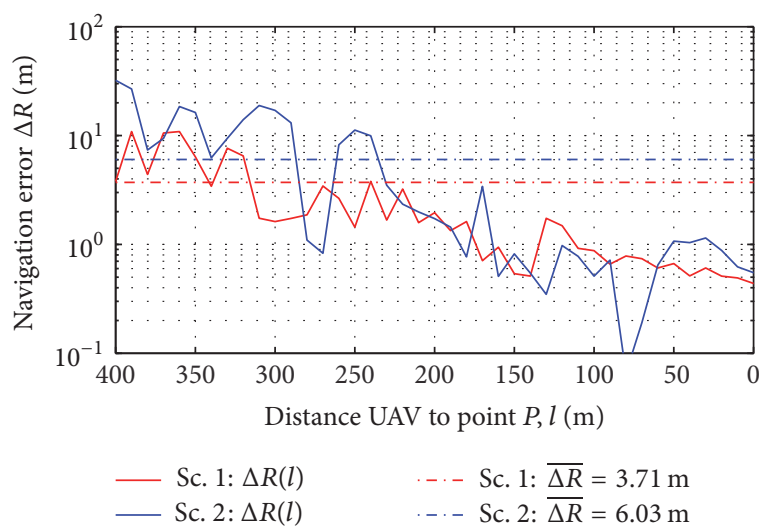

FIgURE 6: Navigation error for Sc. 1 and Sc. 2 and $T_{2}$.

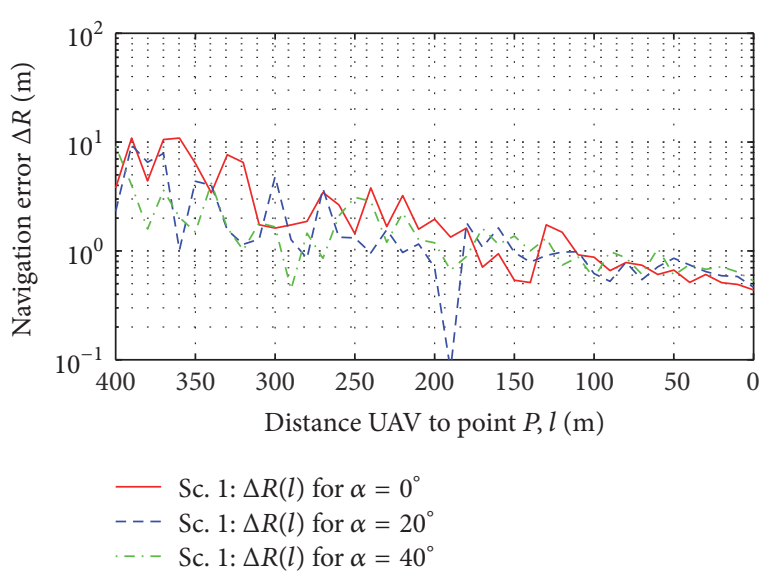

Figure 7: Navigation error for $\alpha=0^{\circ}, \alpha=20^{\circ}$, and $\alpha=40^{\circ}-$ Sc. 1 . 


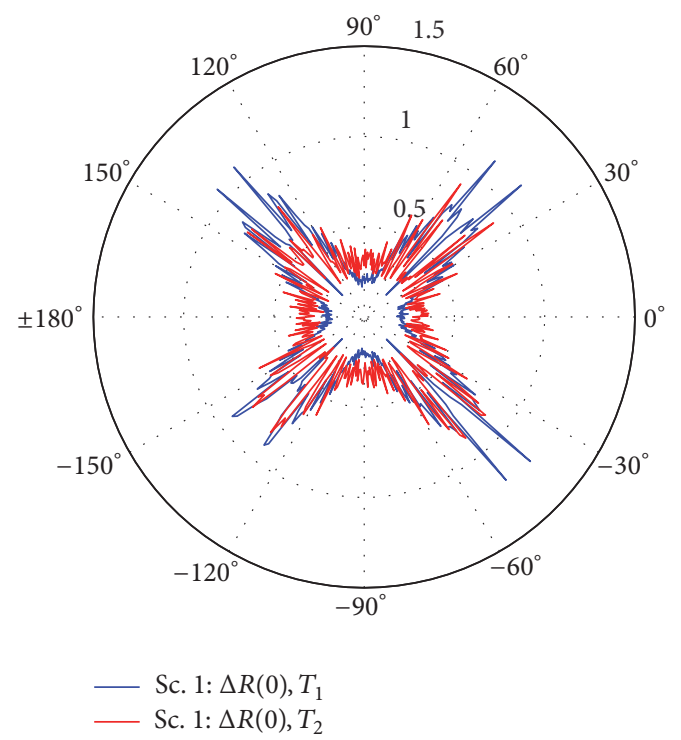

FIGURE 8: Final navigation error versus arrival direction for Sc. 1.

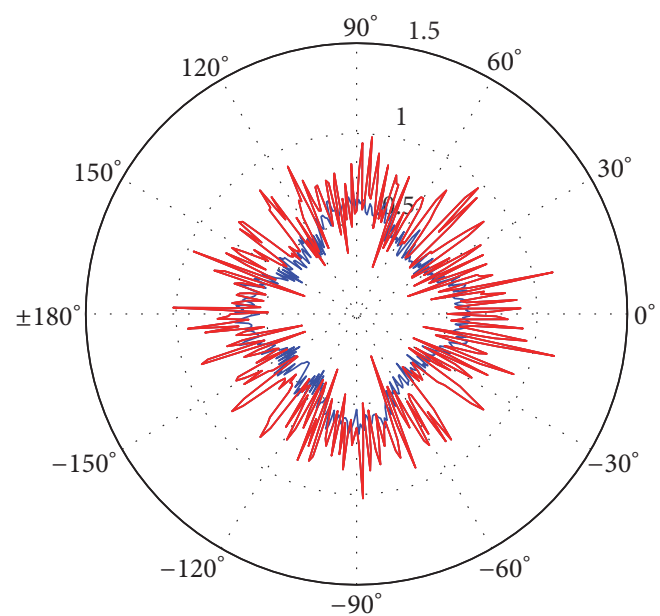

Sc. 2: $\Delta R(0), T_{1}$
Sc. $2: \Delta R(0), T_{2}$

FIGURE 9: Final navigation error versus arrival direction for Sc. 2.

errors. This fact results from the comparison of mean errors, which are $7.10 \mathrm{~m}, 2.66 \mathrm{~m}$, and $3.10 \mathrm{~m}$, respectively, for $\alpha=$ $\left\{0^{\circ}, 20^{\circ}, 40^{\circ}\right\}$.

However, the navigation error that occurs at the destination landing point is most significant. Hence, the influence assessment of the approach direction in the azimuth plane on the final error of the VTOL positioning is made on the basis of simulation research. For Sc. 1 and Sc. 2, the obtained results are presented in Figures 8 and 9, respectively.

For Sc. 1, the obtained results show that the average errors of the final position for all directions are smaller than $0.5 \mathrm{~m}$ for $T_{1}$ and $T_{2}$. In the case of Sc. 2, these errors are $0.52 \mathrm{~m}$ and $0.63 \mathrm{~m}$ for $T_{1}$ and $T_{2}$, respectively. In Figure 8 , we see that there are four crucial sectors of the VTOL arrival for which $\Delta R$ can reach up to $0.90 \mathrm{~m}$ and $1.3 \mathrm{~m}$ for $T_{1}$ and $T_{2}$, respectively. These cases occur when the approach direction overlap with the directions set by pairs of RBs. In the case of Sc. 2 for $T_{2}$, we can see that a distribution of the final errors has a more uniform character. This is also evidenced by the error deviations, which for Sc. 1 are larger $(0.22 \mathrm{~m})$ than for Sc. $2(0.08 \mathrm{~m})$. For $T_{1}$, the error deviations are similar, that is, $0.14 \mathrm{~m}$ and $0.16 \mathrm{~m}$ for Sc. 1 and Sc. 2 , respectively.

The results of the simulation tests show the high positioning accuracy of the aircraft in the landing phase. This indicates the reasonability of the practical implementation of the developed procedure. Based on the results, it can be concluded that the smaller navigation errors were obtained for Sc. 1 and the longer analysis time, that is, for $T_{1}$. However, given the nature of the VTOL flight, the smaller time window may be more practical. In this case, the location of the flight trajectory shown in Sc. 2 may be better, due to the independence of the navigation error from the direction of landing approach.

\section{Conclusions}

This paper provides the navigation procedure that enables the automatic landing approach of VTOL. The developed procedure is based on the Doppler effect and can be made using a simple short-range navigation system that is mounted anywhere at the target UAV landing. Around this place, RBs are deployed, which transmit the harmonic signals and the narrowband signal containing the information about them positions. In addition, the transmission of frequency corrections ensures that the influence of frequency instability of signal sources is minimized. This navigation system can work completely independently of GNSS and requires small spectrum resources.

In the paper, the authors evaluated the impact of the UAV trajectory, the direction of landing approach relative to $\mathrm{RBs}$, and the temporal window of the signal analysis on the accuracy of the developed procedure. This assessment was made on the basis of simulation studies. The results show the high precision of the VTOL positioning. Proper selection of parameters shows that the navigation error near the destination landing point is less than $1 \mathrm{~m}$. The executed research has shown that the developed procedure can contribute to the full automation of the UAV landing process, which may be important for their use in transport.

\section{Conflicts of Interest}

The authors declare that they have no conflicts of interest.

\section{References}

[1] M. Tortonesi, C. Stefanelli, E. Benvegnu, K. Ford, N. Suri, and M. Linderman, "Multiple-UAV coordination and communications in tactical edge networks," IEEE Communications Magazine, vol. 50, no. 10, pp. 48-55, 2012.

[2] M. A. Ma'Sum, M. K. Arrofi, G. Jati et al., "Simulation of intelligent Unmanned Aerial Vehicle (UAV) for military surveillance," in Proceedings of the 2013 5th International Conference on 
Advanced Computer Science and Information Systems, ICACSIS 2013, pp. 161-166, September 2013.

[3] D. Orfanus, E. P. De Freitas, and F. Eliassen, "Self-Organization as a Supporting Paradigm for Military UAV Relay Networks," IEEE Communications Letters, vol. 20, no. 4, pp. 804-807, 2016.

[4] J. A. J. Berni, P. J. Zarco-Tejada, L. Suárez, and E. Fereres, "Thermal and narrowband multispectral remote sensing for vegetation monitoring from an unmanned aerial vehicle," IEEE Transactions on Geoscience and Remote Sensing, vol. 47, no. 3, pp. 722-738, 2009.

[5] J. Wijitdechakul, S. Sasaki, Y. Kiyoki, and C. Koopipat, "UAVbased multispectral image analysis system with semantic computing for agricultural health conditions monitoring and realtime management," in Proceedings of the 2016 International Electronics Symposium (IES), pp. 459-464, Denpasar, Indonesia, September 2016.

[6] P. B. Quater, F. Grimaccia, S. Leva, M. Mussetta, and M. Aghaei, "Light Unmanned Aerial Vehicles (UAVs) for cooperative inspection of PV plants," IEEE Journal of Photovoltaics, vol. 4, no. 4, pp. 1107-1113, 2014.

[7] F. Grimaccia, M. Aghaei, M. Mussetta, S. Leva, and P. B. Quater, "Planning for PV plant performance monitoring by means of unmanned aerial systems (UAS)," International Journal of Energy and Environmental Engineering, vol. 6, no. 1, pp. 47-54, 2015.

[8] S. Daliento, A. Chouder, P. Guerriero et al., "Monitoring, diagnosis, and power forecasting for photovoltaic fields: a review," International Journal of Photoenergy, vol. 2017, Article ID 1356851, 13 pages, 2017.

[9] S. Babak, M. Myslovych, and R. Sysak, "Module structure of UAV-based computerized systems for remote environment monitoring of energy facilities," in Proceedings of the 17th International Conference Computational Problems of Electrical Engineering, CPEE 2016, September 2016.

[10] Y. Lu, D. Macias, Z. S. Dean, N. R. Kreger, and P. K. Wong, "A UAV-Mounted Whole Cell Biosensor System for Environmental Monitoring Applications," IEEE Transactions on Nanobioscience, vol. 14, no. 8, pp. 811-817, 2015.

[11] D. Erdos, A. Erdos, and S. E. Watkins, "An experimental UAV system for search and rescue challenge," IEEE Aerospace and Electronic Systems Magazine, vol. 28, no. 5, pp. 32-37, 2013.

[12] K. A. Ghamry, M. A. Kamel, and Y. Zhang, "Cooperative forest monitoring and fire detection using a team of UAVs-UGVs," in Proceedings of the 2016 International Conference on Unmanned Aircraft Systems, ICUAS 2016, pp. 1206-1211, June 2016.

[13] C. Yuan, Z. Liu, and Y. Zhang, "Vision-based forest fire detection in aerial images for firefighting using UAVs," in Proceedings of the 2016 International Conference on Unmanned Aircraft Systems, ICUAS 2016, pp. 1200-1205, June 2016.

[14] S. Rathinam, P. Almeida, Z. Kim et al., "Autonomous searching and tracking of a river using an UAV," in Proceedings of the 2007 American Control Conference, ACC, pp. 359-364, July 2007.

[15] D. Popescu, L. Ichim, and T. Caramihale, "Flood areas detection based on UAV surveillance system," in Proceedings of the 19th International Conference on System Theory, Control and Computing, ICSTCC 2015 - Joint Conference SINTES 19, SACCS 15, SIMSIS 19, pp. 753-758, October 2015.

[16] UNICEF website, https://www.unicef.org/.

[17] AirVein, 11-Aug-], http://www.airvein.pl/.

[18] G. Hoareau, J. J. Liebenberg, J. G. Musial, and T. R. Whitman, "Package transport by unmanned aerial vehicles," US0068265 A1, 2016.
[19] P. D. Groves, Principles of GNSS, inertial, and multisensor integrated navigation systems, Artech House, Boston, MA, USA, 2nd edition, 2013.

[20] M. S. Grewal, A. P. Andrews, and C. G. Bartone Wiley-Interscience, Hoboken, 3rd edition, 2013.

[21] E. D. Kaplan, Understanding GPS: Principles and applications, Artech House, Boston, MA, USA, 2nd edition, 2005.

[22] F. van Diggelen, A-GPS: Assisted GPS, GNSS, and SBAS, Artech House, 2009.

[23] S. Ward, J. Hensler, B. Alsalam, and L. F. Gonzalez, "Autonomous UAVs wildlife detection using thermal imaging, predictive navigation and computer vision," in Proceedings of the 2016 IEEE Aerospace Conference, AERO 2016, March 2016.

[24] F. S. Leira, T. A. Johansen, and T. I. Fossen, "Automatic detection, classification and tracking of objects in the ocean surface from UAV s using a thermal camera," in Proceedings of the 2015 IEEE Aerospace Conference, AERO 2015, March 2015.

[25] H. Sheng, H. Chao, C. Coopmans, J. Han, M. McKee, and Y. Chen, "Low-cost UAV-based thermal infrared remote sensing: Platform, calibration and applications," in Proceedings of the 2010 IEEE/ASME International Conference on Mechatronic and Embedded Systems and Applications, MESA 2010, pp. 38-43, July 2010.

[26] C. Leśnik, P. Serafin, and A. Kawalec, "Azimuth ambiguity suppression in SAR images using Doppler-sensitive signals," Bulletin of the Polish Academy of Sciences: Technical Sciences, vol. 63, no. 1, pp. 221-227, 2015.

[27] B. Dawidowicz, A. Gadoś, A. Gorzelańczyk et al., "First polish SAR trials," IEE Proceedings: Radar, Sonar and Navigation, vol. 153, no. 2, pp. 135-143, 2006.

[28] M. Caris, S. Stanko, M. Malanowski et al., "Mm-Wave SAR demonstrator as a test bed for advanced solutions in microwave imaging," IEEE Aerospace and Electronic Systems Magazine, vol. 29, no. 7, pp. 8-15, 2014.

[29] L. J. Ippolito, "Satellite Communications Systems Engineering: Atmospheric Effects, Satellite Link Design and System Performance," Satellite Communications Systems Engineering: Atmospheric Effects, Satellite Link Design and System Performance, pp. 1-376, 2008.

[30] Instrument flying handbook, CreateSpace Independent Publishing Platform, 2013.

[31] M. Kayton and W. R. Fried, Avionics Navigation Systems, John Wiley \& Sons, Inc., Hoboken, NJ, USA, 1997.

[32] J. Rafa and C. Ziółkowski, "Influence of transmitter motion on received signal parameters - Analysis of the Doppler effect," Wave Motion, vol. 45, no. 3, pp. 178-190, 2008.

[33] J. M. Kelner, Analizadopplerowskiej methodlokalizacjizródelemisjifalradiowych, rozprawadoktorska (Analysis of the Doppler location method of the radio waves emission sources, Military University of Technology, 2010.

[34] P. Gajewski, C. Ziółkowski, and J. M. Kelner, "Using SDF method for simultaneous location of multiple radio transmitters," in Proceedings of the 2012 19th International Conference on Microwaves, Radar and Wireless Communications, MIKON 2012, pp. 634-637, May 2012.

[35] A. T. de Hoop, "Comments on: J. Rafa, C. Ziólkowski, "Influence of transmitter motion on received signal parametersan analysis of the Doppler effect", Wave Motion 45 (2008) 178-190 [MR2449241]," Wave Motion. An International Journal Reporting Research on Wave Phenomena, vol. 46, no. 1, pp. 8991, 2009. 
[36] N. A. Lehtomaki, T. C. Poling, and D. A. Schuyler, "Single platform Doppler geolocation,” Article ID 130794, pp. 03-2015, 2015.

[37] J. M. Kelner, C. Ziółkowski, and L. Nowosielski, "Local navigation system for VTOLs used on the vessels," in Proceedings of the IEEE/ION Position, Location and Navigation Symposium, PLANS 2016, pp. 415-421, April 2016.

[38] J. M. Kelner, C. Ziółkowski, L. Nowosielski, and M. Wnuk, "Reserve navigation system for ships based on coastal radio beacons," in Proceedings of the IEEE/ION Position, Location and Navigation Symposium, PLANS 2016, pp. 393-402, April 2016.

[39] J. M. Kelner, C. Ziółkowski, and P. Marszalek, "Influence of the frequency stability on the emitter position in SDF method," in Proceedings of the 2016 International Conference on Military Communications and Information Systems, ICMCIS 2016, May 2016.

[40] J. M. Kelner and C. Ziółkowski, "The use of SDF technology to BPSK and QPSK emission sources' location," Przeglad Elektrotechniczny, vol. 91, no. 3, pp. 61-65, 2015. 


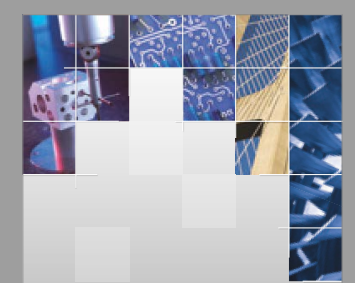

\section{Enfincering}
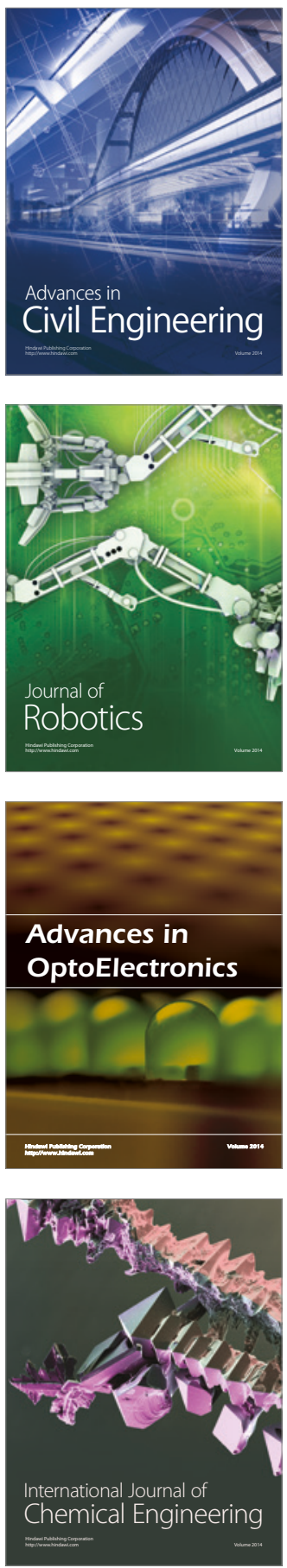

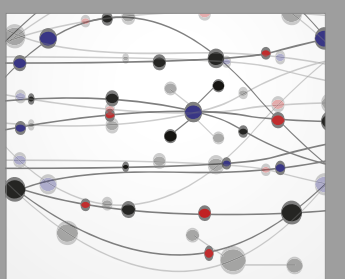

The Scientific World Journal

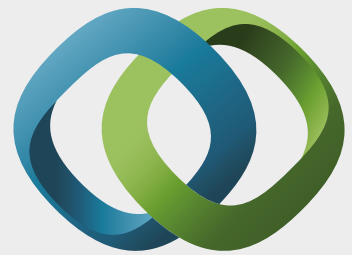

\section{Hindawi}

Submit your manuscripts at

https://www.hindawi.com
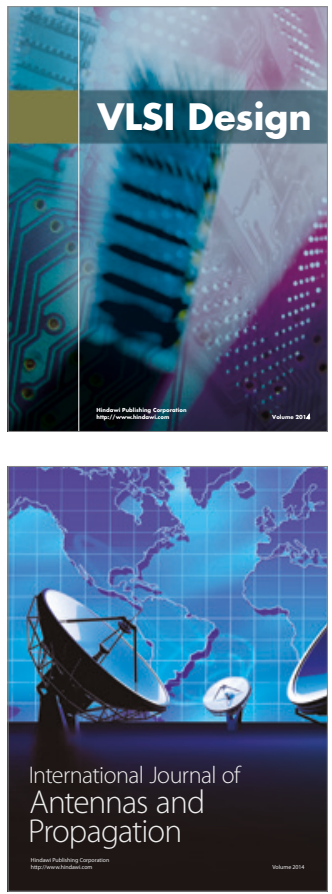

\section{Rotating}

Machinery
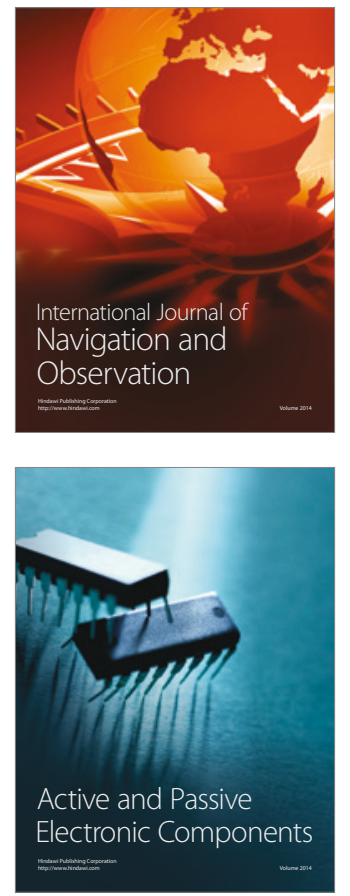
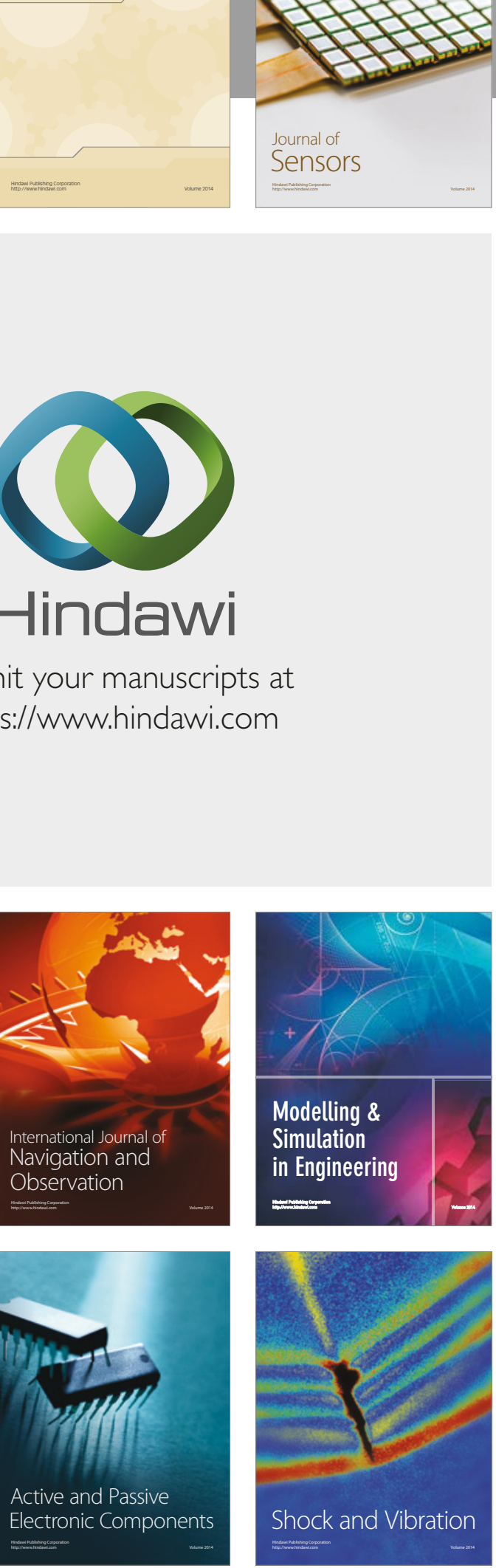
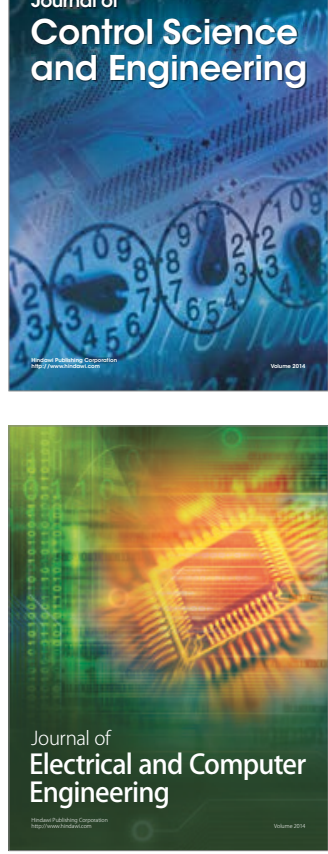

Distributed

Journal of

Control Science

and Engineering
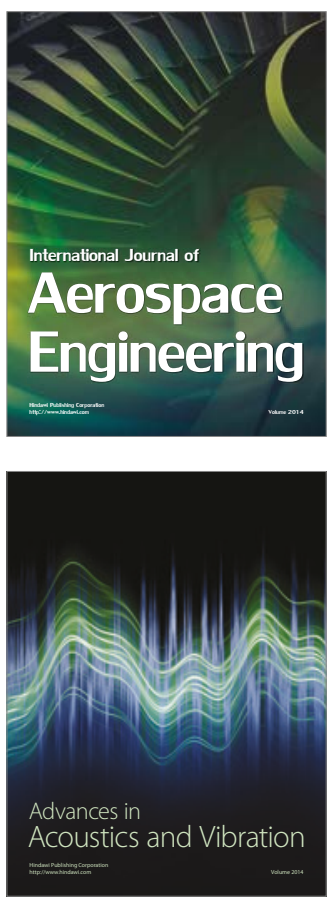

Sensor Networks 